\title{
The Nonlinear Phenomena of Thin Polydimethylsiloxane (PDMS) Films in Electrowetting
}

\author{
Wei Dai and Ya-Pu Zhao* \\ State Key Laboratory of Nonlinear Mechanics (LNM), Institute of Mechanics, Chinese \\ Academy of Sciences, Beijing 100080, China
}

\begin{abstract}
Electrowetting (EW) is an effective way to manipulate small volume liquid in micro- and nano-devices, for it can improve its wettability. Since the late 1990s, electrowetting-on-dielectric (EWOD) has been used widely in bio-MEMS, lab-on-a-chip, etc. Polydimethlsiloxane (PDMS) is extensively utilized as base materials in the fabrication of biomedical micro- and nano-devices. The properties of thin PDMS films used as dielectric layer in EW are studied in this paper. The experimental results show that the thin PDMS films exhibit good properties in EWOD. As to PDMS films with different thicknesses, a threshold voltage and a hysteresis were observed in the EWOD experiments.
\end{abstract}

Keywords: Electrowetting, electrowetting-on-dielectric, Polydimethlsiloxane (PDMS), contact angle, Lippmann-Young equation

The objective of Science is "to measure what is measurable and make measurable what is not".

—Galileo Galilei (1564-1642)

\section{Introduction}

Electrowetting (EW) is a well known method for improving wettability of surfaces, because of the large change of the contact angle for liquid droplet resulted from external electric field [1]. One advantage of EW is that hydrophilicity can be obtained without changing the chemical composition of a system. Additionally, the electrical control of the microfluidic motion is highly promising for microdevices and easy manipulations. Researches in the field of EW have attracted much interest. In fact, the mechanism of EW is similar to that of electrospinning [2-6].

\footnotetext{
* Corresponding author, Email: yzhao@imech.ac.cn (Y.P
} Zhao)
The most common configuration for experiments and practical applications in EW is that a typical dielectric layer (insulator film) of micrometer thickness separates the metal electrode from the electrolyte droplet. This popular experimental method is called EWOD, and is sketched in Fig. 1.

In the current research about EWOD, the phenomena on different dielectric materials are studied [7-10], and the applications in micro-devices are developed fast [11-15]. The interpretation about the principle of EW also attracts much attention [16-18].

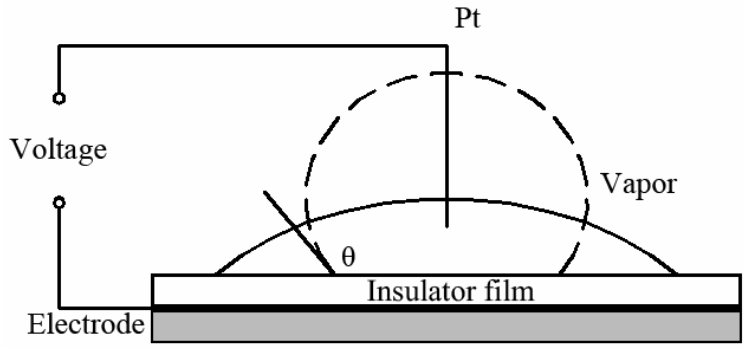

Figure 1. EW behavior of a conductive liquid on an insulator film. The external voltage is applied between a thin Pt wine electrode and a well conductive membrane on the glass. 
Polydimethylsiloxane (PDMS) is widely used as a base material for bio-MEMS/NEMS devices $[19,20]$. The well-known advantages of PDMS include: it is optically transparent, chemically inert, non-toxic and non-flammable. However, the disadvantage of the PDMS is that the surface of PDMS is inherently hydrophobic, and it is difficult to transfer and spread aqueous solutions [21]. A number of techniques have been utilized to hydrophilize the PDMS surface, including physical method, chemical method or a combination of both. Nevertheless, these methods do not satisfy the demands, because of the quick hydrophobic recovery, the complicated process or the chemical hangover [22-25].

The objective of this paper is to study the properties of the PDMS films in EWOD. PDMS films of different thicknesses are fabricated. The EW effects on these films are prominent, and the phenomena influenced by the storage of electrostatic energy in PDMS are also found.

\section{Theory}

The contact angle $\theta$ of a sessile droplet on the surface is calculated by the Young's equation [26], deriving from the force equilibrium at the horizontal direction by the surface tensions in the solid-vapor $\left(\gamma_{s v}\right)$, liquid-vapor $\left(\gamma_{l v}\right)$ and solid-liquid $\left(\gamma_{s l}\right)$ interfaces:

$$
\gamma_{l v} \cos \theta=\gamma_{\mathrm{sv}}-\gamma_{\mathrm{sl}} .
$$

Through the study of Lippmann's experiment about the interface between the electrolyte solution and hydrargyrum in $1875, \gamma_{s l}$ is a function of the electrode potential $U$, which is well known as the Lippmann's equation $[27,28]$

$$
\gamma_{s l}=\gamma_{s l 0}-\frac{1}{2} C_{H}\left(U-U_{P Z C}\right)^{2},
$$

where $U_{P Z C}$ is the potential of zero charge, $\gamma_{s l 0}$ the surface tension with no voltage application, and $C_{H}$ the capacitance of the electric double layer (EDL) [10,29]. When Eq. (2) is inserted into Eq. (1), the contact angle change of the droplet placed directly on an electrode is

$$
\cos \theta_{e}=\cos \theta_{0}+\frac{1}{2} \frac{C_{H}}{\gamma_{l v}}\left(U-U_{P Z C}\right)^{2} .
$$

However, the EW on conductive surfaces has the limitation of the electron transfer from the electrode to redox-active species in the liquid [10], so the EWOD for the practical applications is used generally. At this case, the system can be described as two capacitors in series, composed by the capacitances of EDL $C_{H}$ and the dielectric layer $C_{d}$. The order of EDL is at the Debye length with the typical value at nano-scale in electrolyte. The thickness of the dielectric layer is at micro-scale. So the dielectric layer thickness is usually much larger than the Debye length, and $C_{d}<<C_{H}$, the total capacitance $C \approx C_{d}$. For a simple planar surface per unit area, the capacitance $C_{d}=\varepsilon \varepsilon_{0} / d$, here $d$ is the thickness of the dielectric layer, $\varepsilon$ and $\varepsilon_{0}$ are the dielectric constants of the insulator film and vacuum, respectively. As the spontaneous adsorption of charge at the dielectric layer is small, $U_{P Z C}$ is set to be zero. Then, the surface tension at the dielectric layer surface with the applied voltage is [29]

$$
\gamma_{s l}^{e l f}=\gamma_{s l 0}-\frac{1}{2} \frac{\varepsilon \varepsilon_{0} U^{2}}{d}
$$

where $\gamma_{s l}^{\text {eff }}$ is the effective solid-liquid surface tension, because the dielectric layer is considered as part of the solid-liquid interface. The effect to the solid-liquid surface tension containing the dielectric layer is different from EDL. The electric potential at the EDL influences the solid-liquid surface tension directly. When we substitute Eq. (4) into Eq. (1), the basic EW equation, which is called Lippmann-Young equation, is obtained for EWOD [29]:

$$
\cos \theta_{e}=\cos \theta_{0}+\eta,
$$

where $\eta=\varepsilon \varepsilon_{0} U^{2} /\left(2 d \gamma_{l v}\right)$ is the dimensionless EW number, measuring the ratio between electrostatic energy per unit area and the surface tension.

In many EW experiments, the validity of the Lippmann-Young equation (Eq. (5)) is at the voltage which is not too high $[29,30]$. The 
contact angle change is small or the contact angle does not decrease, when the voltage is over a critical voltage. This phenomenon is called the contact angle saturation [31,32]. The "modified" Lippmann's equation [30] is used to fit the contact angle change from low to high voltage,

$$
\frac{\cos \theta-\cos \theta_{0}}{\cos \theta_{s}-\cos \theta_{0}}=L\left(\frac{C U^{2}}{2 \gamma_{l v}\left(\cos \theta_{s}-\cos \theta_{0}\right)}\right),
$$

where $\theta_{s}$ is the contact angle at saturation, $L$ is the Langevin's function $L(x)=\operatorname{coth}(3 x)-1 / 3 x$. Eq.(6) is more suitable for the EW at saturation.

The voltage can not be loaded very high in our experiments, because the breakdown voltages of the thin PDMS films are less than the critical voltages when the saturation occurs. So the Lippmann-Young equation is adapted in our experiments.

\section{Experimental}

\subsection{Preparation of the thin PDMS films}

The PDMS films were fabricated directly on the indium-tin-oxide (ITO) glass with the plates of $40 \mathrm{~mm} \times 40 \mathrm{~mm} \times 1.1 \mathrm{~mm}$, on which the ITO layer was used as the electrode. The ITO glass must be cleaned carefully before the PDMS film was coated on it. The sonicleaning was carried out by thorough rinsing in high purity water, reagent acetone and ethanol for 5 minutes, respectively. When the plates were dry, the PDMS films (Sylgard 184, Dow Corning, USA; ratio of the base to curing agent $=10: 1$ ) were spread onto the ITO layer by spin coating. The PDMS material was vacuumed for ten minutes to remove the trapped air-bubbles beforehand. Films of different thicknesses were obtained by adjusting the rotation frequency of the spin coater (KW-4A). Then the PDMS films were dried in air at $75^{\circ} \mathrm{C}$ for about 4 hours.

\subsection{Experimental details}

The contact angles were measured by the OCA20 system from Dataphysics, Germany. The diameter of the Pt electrode inserted into the droplet was $0.1 \mathrm{~mm}$. A dc voltage was applied using the Power Regulator (WWL-LDG51). Droplets of deionized water with $0.1 \mathrm{M} \mathrm{KCl}$ were deposited to the film from a syringe with a diameter less than $2.7 \mathrm{~mm}$, which was the capillary length [33] of water. In present work, the effect of the gravity was neglected in our experiments. The contact angles were measured in the voltage cycle from $0 \mathrm{~V}$ to the voltage just below the breakdown voltage of the PDMS film and back to $0 \mathrm{~V}$. The increasing step was $20 \mathrm{~V}$ and the decreasing step was $40 \mathrm{~V}$. The time for a cycle was about 4 minutes.

\section{Results and discussion}

The thicknesses of the PDMS films were measured by the Surface Profiler (Dektek II A). The relationship between the rotating speed of the spin coater and the thickness is shown in Fig. 2. Every data in the figure is the average value of three points chosen randomly. The thickness is decreased from tens of micrometers to several micrometers with the increasing rotating speed, and the thickness changes little when the speed is over 4500 circle/minute.

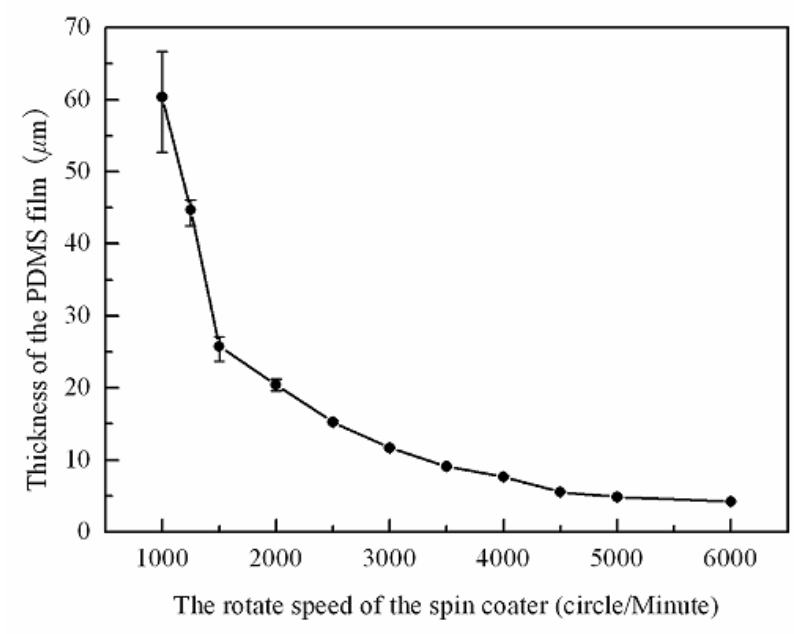

Figure 2. Relationship between the film thickness and the rotating speed.

According to previous researches, surface roughness can influence the contact angle [34]. The contact angle on rough surface is different from that on smooth surface. This relationship is described by the Wenzel equation [35], $\cos \theta=R \cos \theta_{0}$ where $R$ is the roughness factor, giving the ratio of actual to projected 
solid-liquid contact area. Thus, the surface morphology of the PDMS film was investigated by atomic force microscopy (AFM, VEECO, Co. USA). The AFM images for the PDMS films of $60.4 \mu \mathrm{m}$ and $44.7 \mu \mathrm{m}$ thickness are shown in Figs. 3 and 4, respectively. The scanning region was in a square of $5 \mu \mathrm{m} \times 5 \mu \mathrm{m}$. The surface images for films of other thickness were not obtained, because the PDMS surfaces were too sticky to be tested. From the experimental results, the surface relief is less than $10 \mathrm{~nm}$, which is very small compared with the diameter of the droplet. The EW experiments are regarded as on smooth PDMS surfaces.

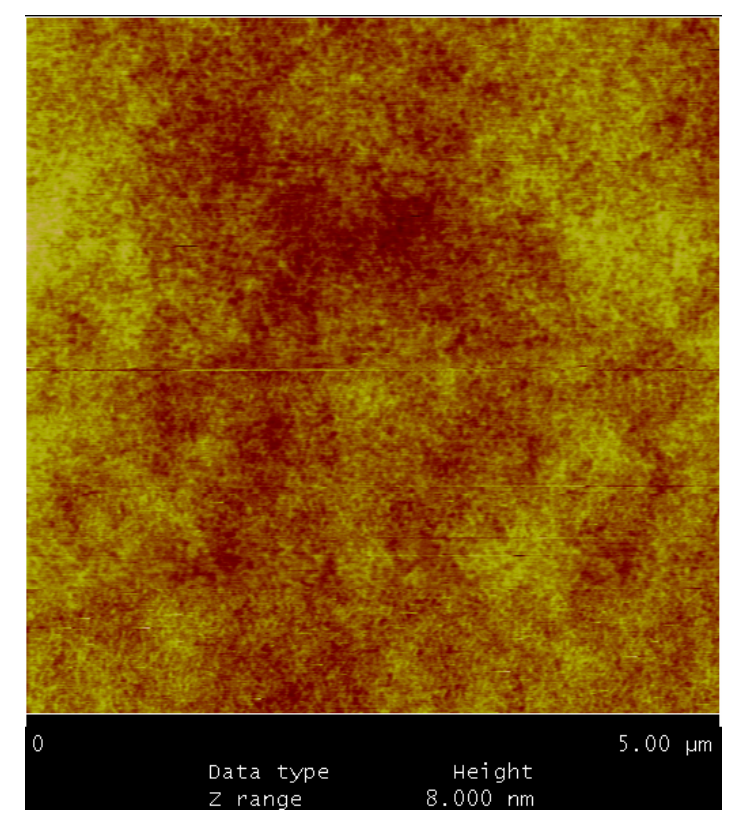

Figure 3. AFM image of the $60.4 \mu \mathrm{m}$ PDMS film (Data types are height).

Figure 5 exhibits the images of a droplet on the PDMS film of $9.1 \mu \mathrm{m}$ thickness. The upper image shows the contact angle when the droplet was freshly deposited on the PDMS film. The middle image shows the contact angle at the maximum voltage $360 \mathrm{~V}$. The bottom image shows the contact angle when the voltage was reduced to zero. The contact angles in the three images are $110.1^{\circ}, 46.9^{\circ}$, and $104.6^{\circ}$, respectively. The decrease of the contact angle is $63.2^{\circ}$, so the effect of EW on this thin PDMS film is remarkable. The reversibility $[36,37]$ of the EW process is very good because the contact angle almost turns back to the initial one, when the voltage is removed.

In these experiments, the breakdown [8] of the PDMS film was observed. This phenomenon occurred frequently when the film was thinner than $5 \mu \mathrm{m}$. Bubbles were produced by the electrolysis and the PDMS films were destroyed in varying degrees, as shown in Fig. 6.

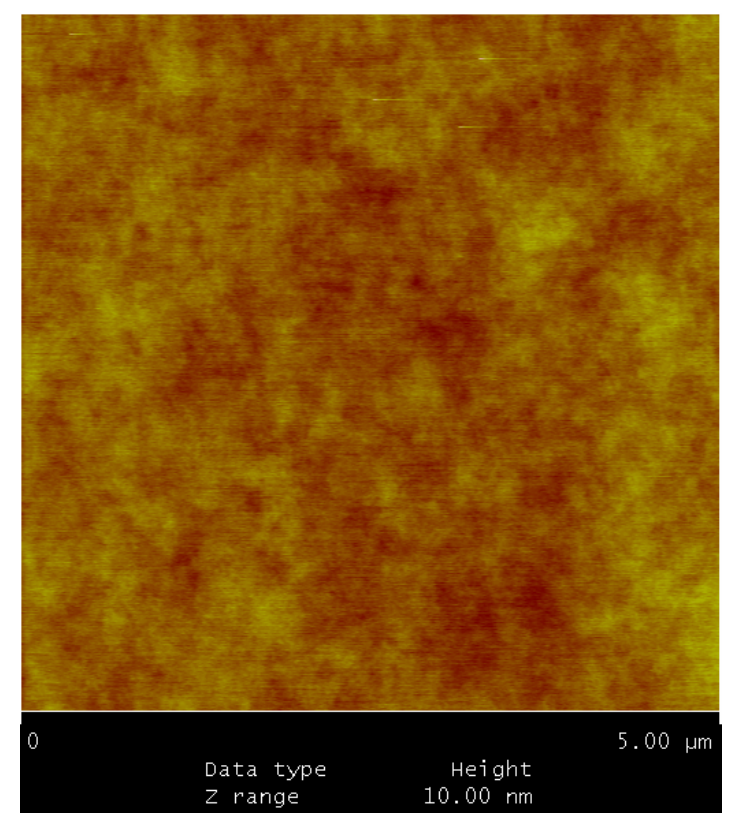

Figure 4. AFM image of the $44.7 \mu \mathrm{m}$ PDMS film (Data types are height).

Figure 7 shows the variation of contact angle with the applied voltage on PDMS films of various thicknesses. The average value of 3-5 groups of experimental results was adopted as the contact angle. During the EW experiments, the contact angle change on the PDMS films of tens of micrometers in thickness is more than $30^{\circ}$, and the wettability converts from hydrophobic to hydrophilic. The contact angle change increases with the thinning of the films, and the largest value can reach more than $60^{\circ}$. As it is pointed out previously, the breakdown phenomena happened frequently when the film was thinner than $5 \mu \mathrm{m}$. The thinnest film in these EW experiments was $7.7 \mu \mathrm{m}$. The reversibility is also visible on these PDMS films of different thicknesses, as all the contact angles come back to the hydrophobic situations. 

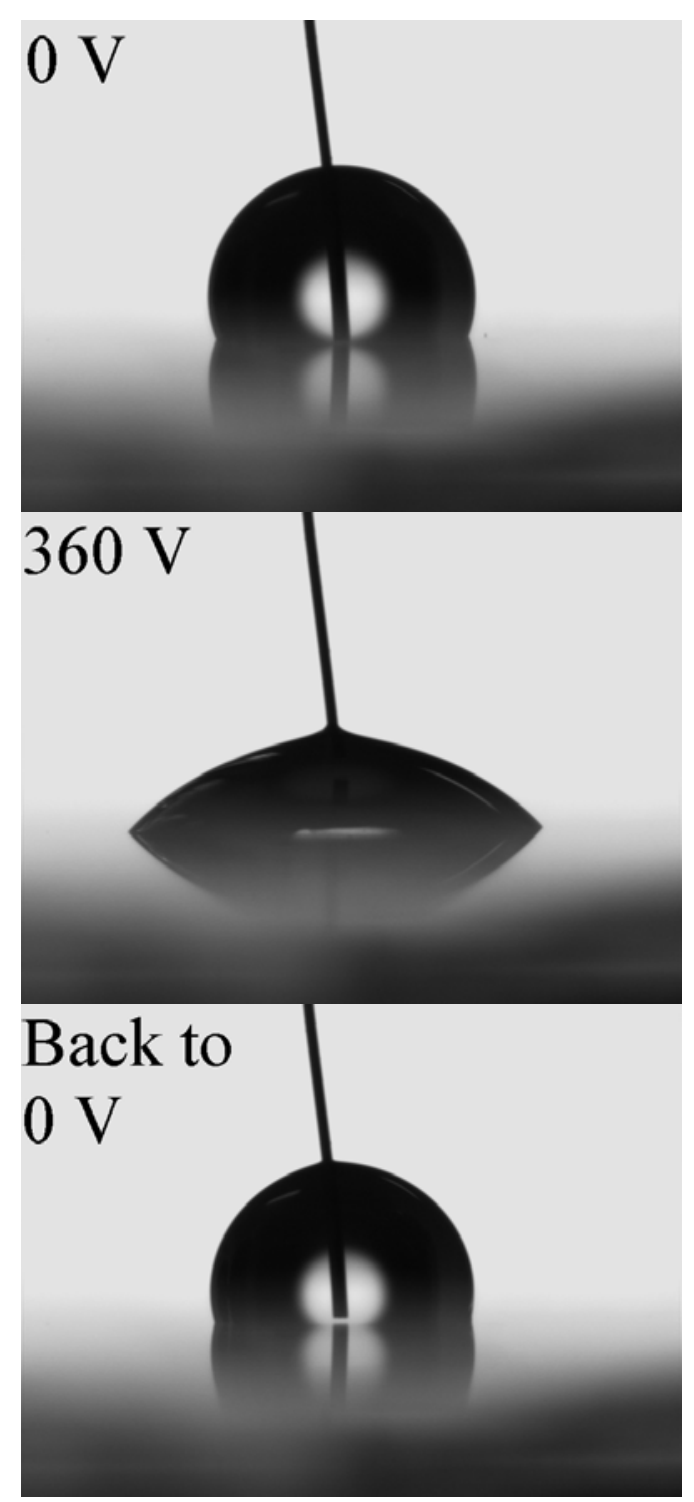

Figure 5. Images of the droplet on the $9.1 \mu \mathrm{m}$ PDMS film at $0 \mathrm{~V}, 360 \mathrm{~V}$, and back to $0 \mathrm{~V}$.

In Fig. 8, we show the cosine of the contact angle as a function of the square of the applied voltage. It is clear that there is a threshold voltage $U_{0}$ when the voltage is increasing. Before this threshold voltage, the change of the contact angle is very small, and after this threshold voltage, the contact angle changes obviously with the increasing voltage. If this threshold voltage is introduced in the Lippmann-Young equation, we can obtain an equation when the voltage exceeds this threshold voltage:

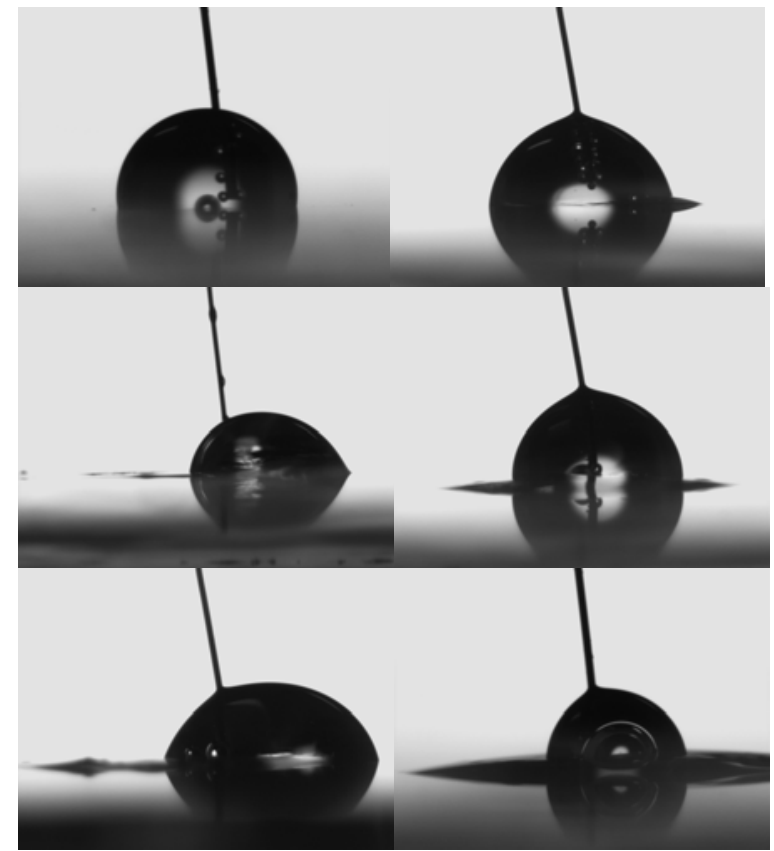

Figure 6. Bubbles were produced in the electrolysis and the PDMS films were destroyed when the breakdown happened.

$$
\cos \theta_{e}=\cos \theta_{0}+\frac{\varepsilon \varepsilon_{0}}{2 d \gamma_{l v}}\left(U-U_{0}\right)^{2},\left(U \geq U_{0}\right) .(7)
$$

From Fig. 8 we can clearly see that threshold voltage decreases as the PDMS film becomes thinner. In Fig. 9, the relationship between the thicknesses of the PDMS films and the threshold voltages is exhibited, and there is an approximately linear relationship between them. This threshold voltage might be determined by the electrostatic energy deposited in the films. The thicker the film is, the more electrostatic energy is stored, so the threshold voltage of PDMS film increases with its thickness.

The hysteresis phenomena are also obviously noticed in Fig. 8 when the voltage is decreasing. The hysteresis effect would be weakened if the film became thinner. The reason of these phenomena is also attributed to the deposited electrostatic energy in the films. When the voltage is decreased, the stored energy is released. More energy is needed to release when the film is thicker and the energy released at solid-liquid interface is less, so the change of the contact angle is smaller. 


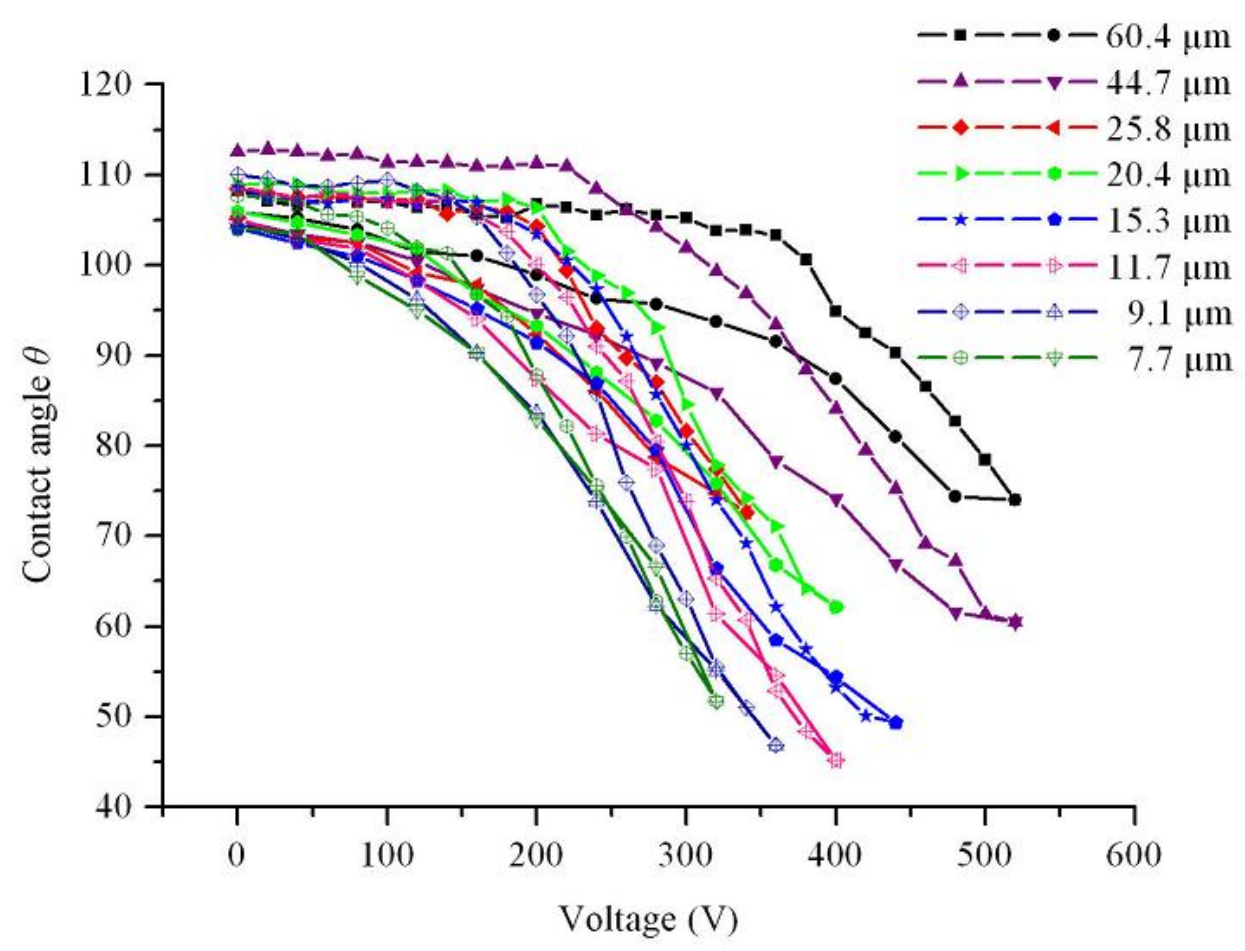

Figure 7. The contact angle versus the voltage on PDMS films of different thickness.

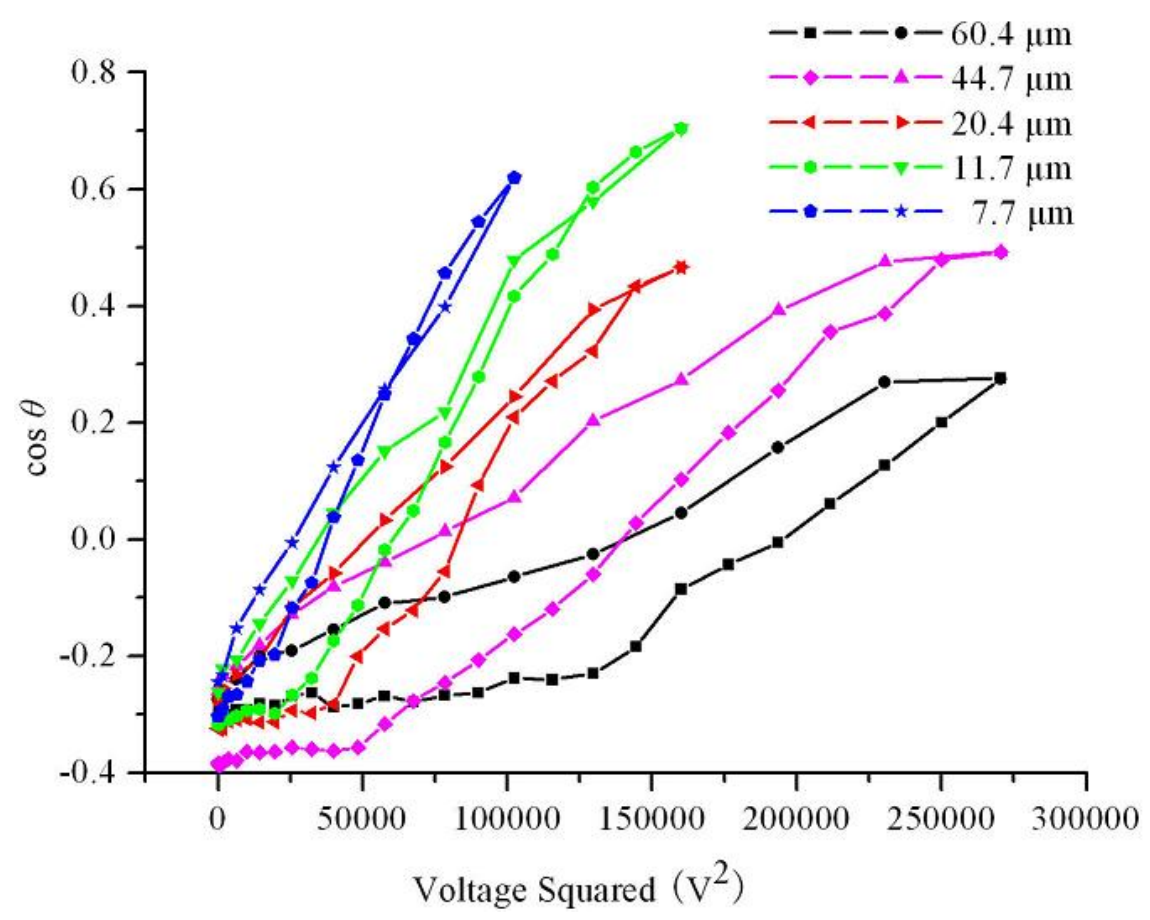

Figure 8. The cosine of the contact angle versus the squared voltage on PDMS films of different thickness. 


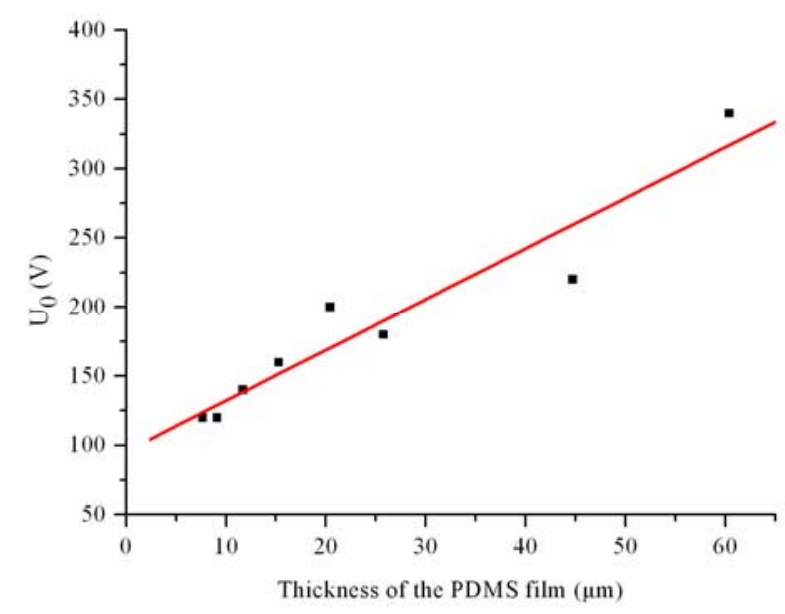

Figure 9. The relationship between the thickness of the PDMS film and the threshold voltage.

\section{Conclusion}

In this paper, EW behaviors of the PDMS films of different thicknesses are studied experimentally. The contact angle is measured while a cyclic voltage is loaded. A large change of the contact angle is observed through EWOD, and the contact angle can almost return to the initial value. When the voltage increases, a threshold voltage is observed. This threshold voltage is approximately linear to the thickness of the PDMS film. When the voltage decreases, the hysteresis occurs. The hysteresis effect is weakened when the film becomes thinner. The determinant factor for these phenomena is the deposited electrostatic energy in PDMS films. A further systematic study of the hysteresis phenomenon in EWOD is needed [38].

\section{Acknowledgments}

This work was supported by the National Basic Research Program of China (973 Program, Grant No. 2007CB310500), NSFC (Grant No. 10225209) as well as key project from CAS (Grant No. KJCX-SW- L2).

\section{References}

[1] Mugele F, et al. Electrowetting: a convenient way to switchable wettability patterns, $J$ Phys-Condens Mat, 17: S559-S576 (2005)

[2] He JH, Liu Y, Xu L, Yu JY. Micro sphere with nanoporosity by electrospinning, Chaos Soliton Fract, 32: 1096-1100 (2007)

[3] $\mathrm{Wu} \mathrm{Y,} \mathrm{Yu} \mathrm{JY,} \mathrm{He} \mathrm{JH,} \mathrm{Wan} \mathrm{YQ.}$ Controlling stability of the electrospun fiber by magnetic field, Chaos Soliton Fract, 32: 5-7 (2007)

[4] $\mathrm{He} J H$, Wan YQ, Xu L. Nano-effects, quantum-like properties in electrospun nanofibers, Chaos Soliton Fract, 33: 26-37 (2007)

[5] Zeng YC, et al. Numerical approach to electrospinning, Int J Nonlinear Sci Numer Simul, 7: 385-388 (2006)

[6] $\mathrm{Xu} \mathrm{L,} \mathrm{He} \mathrm{JH,} \mathrm{Liu} \mathrm{Y.} \mathrm{Electrospun}$ nanoporous spheres with Chinese drug, Int J Nonlinear Sci Numer Simul, 8: 199-202 (2007)

[7] Welters WJJ, Fokkink LGJ. Fast electrically switchable capillary effects, Langmuir, 14: 1535-1538 (1998)

[8] Seyrat E, Hayes RA. Amorphous fluoropolymers as insulators for reversible low-voltage electrowetting, J Appl Phys, 90: 1383-1386 (2001)

[9] Berry S, Kedzierski J, Abedian B. Low voltage electrowetting using thin fluoroploymer films, $J$ Colloid Interf Sci, 303: 517-524 (2006)

[10] Moon H, Cho SK, Garrell RL, Kim CJ. Low voltage electrowetting-on-dielectric, $J$ Appl Phys, 92: 4080-4087 (2002)

[11] Zhao Z, Cui DF, Wang L. Design and microfabrication of biochemical reaction chip, Int J Nonlinear Sci Numer Simul, 3: 219-222 (2002)

[12] $\mathrm{Wu}$ JG, et al. Studies on digital mierofluidic chip for micro-total-analysis systems, Chin J Anal Chem, 34: 10421046 (2006)

[13] Satoh W, Loughran M, Suzuki H. Microfluidic transport based on direct electrowetting, J Appl Phys, 96: 835-841 (2004)

[14] Moon I, Kim J. Using EWOD (electrowetting-on-dielectric) actuation in a micro conveyor system, Sens Actuator 
A-Phys, 130: 537-544 (2006)

[15] Cho SK, Moon HJ, Kim CJ. Creating, transporting, cutting, and merging liquid droplets by electrowetting-based actuation for digital microfluidic circuits, $J$ Microelectromech Syst, 12: 70-80 (2003)

[16] Bienia M, Vallade M, Quilliet C, Mugele F. Electrical-field-induced curvature increase on a drop of conducting liquid, Europhys Lett, 74: 103-109 (2006)

[17] Buehrle J, Herminghaus S, Mugele F. Interface profiles near three-phase contact lines in electric fields, Phys Rev Lett, 91: 086101 (2003)

[18] Kang KH. How electrostatic fields change contact angle in electrowetting, Langmuir, 18: 10318-10322 (2002)

[19] McDonald JC, Whitesides GM. Poly(dimethylsiloxane) as a material for fabricating microfluidic devices, Accounts Chem Res, 35: 491-499 (2002)

[20] Fujii T. PDMS-based microfluidic devices for biomedical applications, Microelectron Eng, 61-2: 907-914 (2002)

[21] Bodas D, Khan-Malek C. Formation of more stable hydrophilic surfaces of PDMS by plasma and chemical treatments, Microelectron Eng, 83: 1277-1279 (2006)

[22] Seo J, Lee LP. Effects on wettability by surfactant accumulation/depletion in bulk polydimethylsiloxane (PDMS), Sens Actuator B-Chem, 119: 192-198 (2006)

[23] Hillborg H, Sandelin M, Gedde UW. Hydrophobic recovery of polydimethylsiloxane after exposure to partial discharges as a function of crosslink density, Polymer, 42: 7349-7362 (2001)

[24] Hillborg H, Gedde UW. Hydrophobicity recovery of polydimethylsiloxane after exposure to corona discharges, Polymer, 39: 1991-1998 (1998)

[25] Bodas D, Khan-Malek C. Hydrophilization and hydrophobic recovery of PDMS by oxygen plasma and chemical treatment--An SEM investigation, Sens Actuator B-Chem, 123: 368-373 (2007)

[26] Young T. An essay on the cohesion of fluids, Philos Trans $R$ Soc London, 95: 65-87 (1805)

[27] Lippmann G. Relations entre les phénomènes electriques et capillaries, Ann Chim Phys, 5: 494-548 (1875)

[28] Antropov LI. Theoretical Electrochemistry, Mir Publishers, Moscow, 1977

[29] Mugele F, Baret JC. Electrowetting: from basics to applications, $J$ Phys-condens Mat, 17: R705-R774 (2005)

[30] Berthier J, Clementz P, Raccurt O, Jary D, Claustre P, Peponnet C, Fouillet Y. Computer aided design of an EWOD microdevice, Sens Actuator A-Phys, 127: 283-294 (2006)

[31] Quinn A, Sedev R, Ralston J. Contact angle saturation in electrowetting, $J$ Phys Chem B, 109: 6268-6275 (2005)

[32] Vallet M, Vallade M, Berge B. Limiting phenomena for the spreading of water on polymer films by electrowetting, Eur Phys J B, 11: 583-591 (1999)

[33] Langbein D. Capillary Surfaces: Shape-Stability-Dynamics, in Particular under Weightlessness, Springer-Verlag, Berlin, 2002

[34] Ren SL, Yang SR, Zhao YP, Xiao XD, Yu TX. Morphology correlation to the superhydrophobicity in an organic thin film on rough $\mathrm{Al}$, Int $J$ Nonlinear Sci Numer Simul, 3: 785-788 (2002)

[35] Wenzel RN. Resistance of solid surfaces to wetting by water, Ind Eng Chem, 28: 988-994 (1936)

[36] Quilliet C, Berge B. Electrowetting: a recent outbreak, Curr Opin Colloid In, 6: 34-39 (2001)

[37] Verheijen HJJ, Prins MWJ. Reversible electrowetting and trapping of charge: Model and experiments, Langmuir, 15: 6616-6620 (1999)

[38] Chao PCP, Liao PY, Huang JS. A novel microscopic modeling on hysteresis effects on piezoelectric structures with assistance from finite element. Int $J$ Nonlinear Sci Numer Simul, 7: 269-274 (2006) 